\title{
ANÁliSE DE PRESCRIÇÃO DE PACIENTES INTERNADOS EM UNIDADE DE TERAPIA INTENSIVA NEONATAL
}

Francineide Ferreira Dantas ${ }^{1}$, Maniele da Mata Lima ${ }^{1}$, Cinthia Cristina Sousa de Menezes da Silveira $^{2}$, Antônio José Cerasi Junior ${ }^{2}$.

${ }^{1}$ Faculdade Cosmopolita, ${ }^{2}$ Fundação Santa Casa de Misericórdia do Pará
E-mail para correspondência: francineidefdantas@ gmail.com Submetido em: 08/06/2021 e aprovado em: 02/08/2021

\begin{abstract}
RESUMO
Introdução: A Unidade de Terapia Intensiva Neonatal (UTIN) é indicada ao atendimento de neonatos que precisem de assistência médica intensiva. Os neonatos apresentam características farmacocinéticas e farmacodinâmicas especiais, ocorrendo mudanças no decorrer de seu desenvolvimento, influenciando particularmente quanto ao uso de medicamentos. Objetivos: Investigar, identificar, classificar e quantificar as ocorrências de Problemas Relacionados aos Medicamentos (PRMs) nas prescrições de pacientes neonatos internados na UTIN da Unidade Infantil Dr. Almir Gabriel da Fundação Santa Casa de Misericórdia do Pará (FSCMP) na cidade de Belém-Pará. Metodologia: Trata-se de um estudo do tipo retrospectivo, descritivo e exploratório, com abordagem quantitativa; os dados coletados referem-se ao período de 01 a 31 de outubro de 2020; para as análises das prescrições foram realizadas pesquisas nas bases de dados Scielo, Pubmed, Micromedex (Pedriatrics, Drug Ref, Interactions, Compatibility, NeoFax), bulas dos medicamentos, manuais e formulários da FSCMP. Resultados e discussão: Foram analisadas 755 prescrições, correspondentes a 80 pacientes hospitalizados nas UTINs. Dentre as prescrições analisadas foram encontrados um total de 925 Problemas Relacionados aos Medicamentos (PRMs), dos quais 30,6\% são referentes à dose, 29,2\% são interações medicamentosas e 20,4\% à via de administração. Conclusões: Assegurar a segurança ao neonato abrange, no geral, o cuidado na prevenção de erros, onde o acompanhamento pelo profissional farmacêutico clínico junto a equipe multiprofissional é de suma importância, garantindo o uso seguro e racional de medicamentos.
\end{abstract}

Palavras-chave: Unidade de Terapia Intensiva Neonatal, Problemas Relacionados aos Medicamentos, Interação Medicamentosa, Atenção Farmacêutica.

\begin{abstract}
Introduction: The Neonatal Intensive Care Unit is indicated for the care of neonates who need intensive medical care. Neonates have special pharmacokinetic and pharmacodynamic characteristics, with changes occurring during their development, particularly influencing the use of medicines. Aims: To investigate, identify, classify and quantify the occurrences of drugrelated problems in the neonates patients prescriptions hospitalized in the Neonatal Intensive Care Unit of the Dr. Almir Gabriel Children's Unit of the Santa Casa de Misericórdia Foundation in the city of Belém-Pará. Methodology: This is a retrospective, descriptive and exploratory study, with a quantitative approach; the data collected refer to the period from 1st to October 31st, 2020; for the analysis of the prescriptions, searches were carried out in the Scielo, Pubmed, Micromedex databases (Pedriatrics, Drug Ref, Interactions, Compatibility,
\end{abstract}


NeoFax), medicine leaflets, manuals and forms of the Santa Casa de Misericórdia do Pará Foundation. Results and discussion: A total of 755 prescriptions were analyzed, corresponding to 80 patients hospitalized in neonatal intensive care units. Among the prescriptions analyzed, a total of 925 drug-related problems were found, of which $30.6 \%$ are related to the dose, $29.2 \%$ are medicaments interactions and $20.4 \%$ to the route of administration. The incidence of medicines questions was 11.56 \pm AVERAGE ERROR. Conclusions: To ensure the safety of the neonates include, in general, the care in the prevention of errors, where the monitoring by the clinical pharmacist with the multidisciplinary team is of paramount importance, ensuring the safe and rational use of medications.

Keywords: Neonatal Intensive Care Unit, Drug-Related Problems, Drug Interaction, Pharmaceutical Attention.

\section{INTRODUÇÃO}

A Unidade de Terapia Intensiva Neonatal (UTIN) é indicada ao atendimento de neonatos, ou seja, recém-nascidos com até 28 dias de vida, e que precisem de assistência médica intensiva. É o local onde esses pacientes contam com auxílio completo e de extrema necessidade para sua recuperação que incluem acompanhamento intenso da equipe multiprofissional, aparelho de ressuscitação, entre outros suportes assistenciais, que aumentam as condições necessárias para a continuação da vida e o crescimento do recém-nascido ${ }^{(1)}$.

O peso, a prematuridade, o índice de $\operatorname{Apgar}\left(1^{\circ}, 5^{\circ}\right.$ e $10^{\circ}$ minuto), são algumas condições de risco que podem estar associado com a ida do neonato a $\operatorname{UTIN}^{(2)}$. Segundo $\operatorname{Costa}^{(3)}$, as características maternas também influenciam diretamente na internação do recém-nascido na UTIN, tais como: raça, idade, gestação múltipla, intervalo interpartal, antecedentes de parto prematuro, de natimorto, de aborto, tipo de parto, além de morbidades como hipertensão, diabetes, infecção urinária, anemia, desnutrição, obesidade, consumo de drogas, bebidas alcoólicas e tabaco.

A quantidade de pesquisas sobre o uso de medicamentos em neonatos tem sido comparativamente menor quando confrontado com as efetuadas nos demais indivíduos. Os neonatos apresentam características farmacocinéticas e farmacodinâmicas especiais, ocorrendo mudanças no decorrer de seu desenvolvimento, influenciando particularmente quanto ao uso de medicamentos $^{(4)}$.

Nos últimos anos, a partir do aumento do interesse, aprendizado e prática entre os profissionais da área da saúde, aconteceram mudanças relevantes no cuidado e acompanhamento dos recém-nascidos, principalmente daqueles que necessitam de assistência à saúde intensivas. Além de pediatras especializados em neonatologia e enfermeiros neonatais, 
foram inseridos na equipe multiprofissional fisioterapeutas, farmacêuticos, nutricionistas, e terapeutas ocupacionais ${ }^{(5)}$.

O Conselho Federal de Farmácia (CFF) recomenda a inserção de, no mínimo, 01(um) farmacêutico clínico para até 15(quinze) pacientes críticos, podendo variar de acordo com a estrutura física e tecnológica de cada instituição e com a complexidade dos pacientes atendidos $^{(6)}$. Entre as funções do farmacêutico clínico nas UTINs estão o acompanhamento da terapêutica medicamentosa a fim de identificar e prevenir Problemas Relacionados aos Medicamentos (PRMs) e resultados negativos associados à medicação. Entende-se como PRM toda condição na qual o procedimento da administração do medicamento provoca ou pode provocar o desenvolvimento de um resultado negativo associado à medicação ${ }^{(7)}$.

As interações medicamentosas são definidas como alteração no efeito de certo fármaco ao ser administrado simultâneo com outro medicamento e/ou alimento. Podem ser classificadas como interação fármaco - fármaco, na administração de dois medicamentos, e fármaco alimento quando há o uso de medicamento com alimento. Essas interações podem ser benéficas, quando há um aumento na eficácia terapêutica, ou danosa quando seus efeitos farmacológicos se elevam de modo que ultrapassem os níveis de segurança, ou quando há diminuição da eficácia ou o surgimento de reações adversas. Estas reações podem ser classificadas como leves, moderadas ou graves, sendo que essa última é considerada como ameaça a vida, além de ser causadora de danos permanentes ao paciente. E quando manifestadas podem ser monitoradas através da farmacovigilância ${ }^{(8,9,10)}$.

Para Organização Mundial da Saúde (OMS), a farmacovigilância é compreendida pela ciência, atividade de identificação, avaliação e prevenção de efeitos adversos ou qualquer problema possível relacionado ao fármaco, os quais melhoram a saúde pública e a segurança em relação ao uso de medicamentos ${ }^{(11)}$. O uso de medicamentos de forma racional deve ser avaliado com maior rigor, principalmente no que tange determinados grupos de pacientes, como por exemplo, imunodeprimidos, pacientes hospitalizados, idosos e crianças. Para esse último, o uso do medicamento deve ser bastante cauteloso, haja vista que esse grupo não apresenta maturação fisiológica completa, portanto, mais vulneráveis aos efeitos colaterais dos medicamentos ${ }^{(12)}$. Essas prevenções devem ser mais ativas dentro do ambiente hospitalar, e com maior intensidade para pacientes internados em Unidades de Terapia Intensiva (UTI), pois normalmente são submetidos ao uso de diversos medicamentos em conjunto. Além desse agravante, muitos desses medicamentos nem sempre são prescritos de forma racional ${ }^{(13,8)}$. 
Considerando as diversas pesquisa realizadas sobre PRMs e a relevância do tema para a segurança dos pacientes neonatos internados em UTIN, observou-se a necessidade de aprofundar a investigação, identificação e classificação das interações medicamentosas, incompatibilidades, erros de doses, entre outros PRMs que foram quantificados em nosso estudo através das prescrições de pacientes neonatos internados na UTIN da Unidade MaternoInfantil Dr. Almir Gabriel da FSCMP.

\section{MÉTODOS}

Realizou-se um estudo do tipo retrospectivo, descritivo e exploratório, com abordagem quantitativa, para avaliar os PRMs na UTIN da FSCMP.

A coleta de dados ocorreu no período de 01 a 31 de fevereiro de 2021, após a autorização do Comitê de Ética e pesquisa da FSCMP, com o parecer de $n^{\circ} 4.522 .247$. Para a pesquisa foram selecionados 40 leitos de UTIN e os dados referentes a esses leitos foram coletados na Gerência de Assistência Farmacêutica e Gerência de informação ao Paciente da FSCMP. Foram analisadas prescrições referentes ao período 01 a 31 de outubro de 2020, assim como as análises das variáveis de estudos relativos aos PRMs como, erros de dose, duplicidade, interação medicamento-medicamento, incompatibilidade, entre outros.

Foram incluídos neste estudo todas as prescrições dos pacientes hospitalizados nas UTIN da FSCMP no período estabelecido. Em relação as prescrições excluídas estavam a de pacientes neonatos encaminhados de outros centros de saúde, dependentes de suporte de vida por tempo indeterminado, com tempo de permanência inferior a 48 horas no setor de estudo, além de pacientes indígenas.

As informações coletadas foram organizadas em planilhas e gráficos do programa Microsoft Office Excel 2019, para melhor visualização dos dados e formulação de gráficos e/ou tabelas com os números absolutos, média aritmética e porcentagens.

Para as análises das prescrições foram realizadas pesquisas nas bases de dados Scielo, Pubmed, Micromedex (Pedriatrics, Drug Ref, Interactions, Compatibility, NeoFax), bulas dos medicamentos, manuais e formulários da FSCMP.

\section{RESULTADOS E DISCUSSÃO}

Foram analisadas 755 prescrições, correspondentes a 80 pacientes hospitalizados na UTIN da FSCMP. Dentre as prescrições analisadas foram encontrados um total de 925 PRMs, 
dos quais 30,6\% são referentes à dose, 29,2\% são interações medicamentosas e 20,4\% à via de administração (Figura 1).

Figura 1: Problemas Relacionados aos Medicamentos encontrados nas prescrições da Unidade de Terapia Intensiva Neonatal

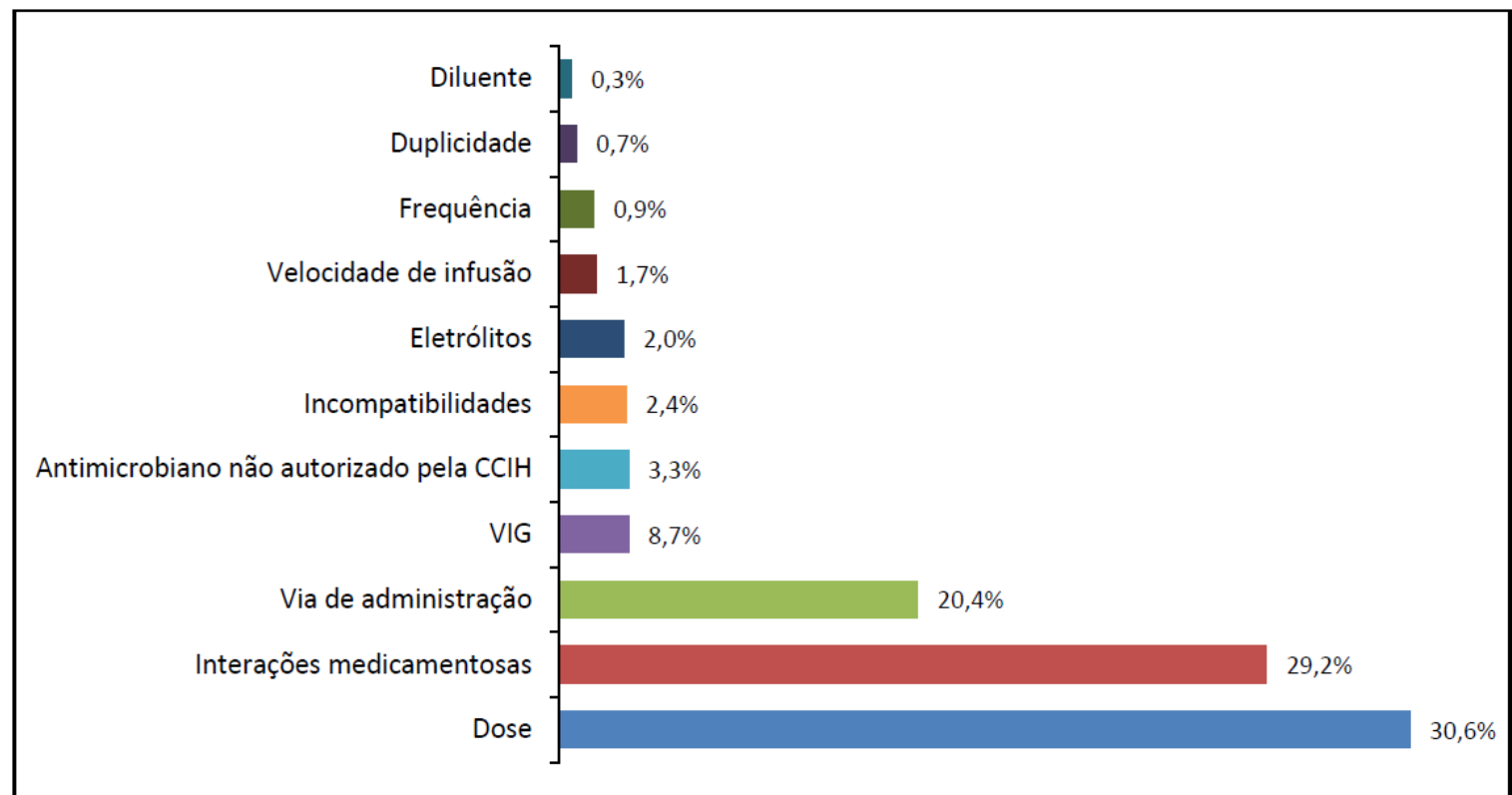

Fonte: Os autores.

De acordo com Leopoldino ${ }^{(14)}$ e Nunes ${ }^{(15)}$ ainda há estudos escassos referentes a análises de PRMs em Neonatos. O cuidado com os pacientes neonatos é muito importante diante de sua peculiaridade fisiológica, a quantidade de medicamentos prescritos e uso de medicamentos classificados como off-label (não licenciados), que pode estar direta ou indiretamente associados aos PRMs ${ }^{(16,17)}$.

Em um estudo de Nascimento ${ }^{(18)} 32,6 \%$ dos PRMs foram interações medicamentosas, corroborando com os resultados encontrados no presente trabalho $(29,2 \%)$.

Os erros de prescrições representam uma grande parcela dos PRMs, que estão geralmente associados com o não seguimento das boas práticas de prescrições, podendo ser observados nas prescrições incompletas que não mencionam via de administração e/ou volume de administração, erros de dose, duplicidade, entre outros. No presente estudo observou-se um número considerável de PRMs, com maior destaque para erros referentes à dose $(30,6 \%)$. Dentre os medicamentos com erros de dose destacou-se a Vitamina A+D (48,0\%) e a Cafeína $(18,6 \%)$ (Tabela 1$)$. 
Tabela 1: Erros de dose encontradas nas prescrições dos pacientes internados nas unidades de terapia intensiva

\begin{tabular}{|c|c|}
\hline Medicamento & $\%$ \\
\hline Vitamina $A+D$ & 58,4 \\
\hline Cafeína & 48,5 \\
\hline Dobutamina & 18,6 \\
\hline Espironolactona & 4,1 \\
\hline Fentanil & 2,9 \\
\hline Fenobarbital & 2,3 \\
\hline Midazolan & 2,3 \\
\hline Meropenem & 2,0 \\
\hline Vancomicina & 2,0 \\
\hline Ampicilina & 1,2 \\
\hline Cefotaxima & 1,2 \\
\hline Ciprofloxacino & 1,2 \\
\hline Hidrocortisona & 1,2 \\
\hline Eletrólitos & 0,9 \\
\hline Furosemida & 0,6 \\
\hline Gentamicina & 0,6 \\
\hline Morfina & 0,6 \\
\hline Oxacilina & 0,6 \\
\hline Ranitidina & 0,6 \\
\hline Epinefrina & 0,3 \\
\hline
\end{tabular}

Fonte: Os autores.

Segundo Nascimento ${ }^{(19)}$ problemas envolvendo doses muito altas ou baixas corresponderam a 30,8\% dos problemas observados. No presente estudo, os problemas relacionados à dose ocorreram na maioria das vezes na primeira prescrição ou na inclusão e/ou troca de medicamento prescrito. Porém, quando a prescrição era avaliada pelo farmacêutico, antes da liberação dos medicamentos, observou-se a correção do erro de dosagem na prescrição por meio da intervenção farmacêutica, ocorrendo o ajuste junto com o prescritor, com a realização de uma nova prescrição, adicionando a dose adequada para os neonatos, de acordo com suas características fisiológicas (peso ou superfície corporal) ${ }^{(20,21,22)}$.

Levando em consideração as características de pacientes neonatos internados em UTI, o risco para ocorrência de interação medicamentosa é maior, diante da complexidade da farmacoterapia empregada, envolvendo o uso simultâneo de diversos fármacos de diferentes classes terapêuticas. As interações medicamentosas foram determinadas e classificadas conforme o encontrado nas principais bases de dados Micromedex Drug Int (Tabela 2). 
Tabela 2. Interações medicamentosas encontradas nas prescrições dos pacientes internados nas Unidades de Terapia Intensiva Neonatal.

\begin{tabular}{|c|c|c|c|c|}
\hline Medicamento 01 & Medicamento 02 & Classificação & $\%$ & Problema \\
\hline Ampicilina & Gentamicina & Leve & 52,8 & Ampicilina diminui a eficácia da gentamicina \\
\hline Furosemida & Insulina & Moderada & 10,5 & Aumento do risco de hiperglicemia \\
\hline Ampicilina & Amicacina & Leve & 8,4 & Ampicilina diminui a eficácia da Amicacina \\
\hline Morfina & Furosemida & Moderada & 8,0 & Morfina diminui o efeito da furosemida \\
\hline Penicilina Cristalina & Gentamicina & Leve & 4,6 & Penicilina diminui a eficácia da gentamicina \\
\hline Morfina & Fenobarbital & Grave & 3,8 & $\begin{array}{l}\text { Risco de depressão respiratória do sistema } \\
\text { nervoso central }\end{array}$ \\
\hline Fentanil & Midazolam & Grave & 3,5 & $\begin{array}{c}\text { Uso de } 2 \text { depressores do SNC resulta em } \\
\text { depressão respiratória, hipotensão, sedação } \\
\text { profunda e morte }\end{array}$ \\
\hline Fentanil & Morfina & Grave & 1,0 & $\begin{array}{l}\text { O uso concomitante da morfina e Fentanil, } \\
\text { pode aumentar em resultado do aumento de } \\
\text { risco da depressão respiratória }\end{array}$ \\
\hline Dexametasona & Fluconazol & Moderada & 1,0 & $\begin{array}{l}\text { O uso concomitante do Fluconazol e } \\
\text { Dexametasona pode resultar em aumento da } \\
\text { exposição ao glicocorticoides e risco de } \\
\text { toxicidade }\end{array}$ \\
\hline Penicilina Cristalina & Amicacina & Leve & 1,0 & Penicilina diminui a eficácia da Amicacina \\
\hline Dexametasona & Fentanil & Grave & 1,0 & $\begin{array}{l}\text { Dexametasona é indutora da CYP3A4 o que } \\
\text { diminui a do Fentanil }\end{array}$ \\
\hline Cloreto de potássio & Espironolactona & Grave & 0,7 & Hipercalemia \\
\hline Furosemida & Amicacina & Grave & 0,7 & Ototoxicidade e nefrotoxicidade \\
\hline Gluc. de cálcio & Ciprofloxacino & Moderada & 0,7 & $\begin{array}{l}\text { Uso concomitante de cálcio, pode diminuir a } \\
\text { eficácia da Ciprofloxacino (quinolonas) }\end{array}$ \\
\hline Ciprofloxacino & Fluconazol & Grave & 0,4 & Prolongamento do intervalo QT \\
\hline Ciprofloxacino & Insulina & Grave & 0,4 & Hipoglicemia ou hiperglicemia \\
\hline Furosemida & Gentamicina & Grave & 0,4 & $\begin{array}{l}\text { O uso concomitante pode resultar no } \\
\text { aumento da concentração plasmática da } \\
\text { gentamicina causando ototoxicidade e } \\
\text { nefrotoxicidade }\end{array}$ \\
\hline Fluconazol & Omeprazol & Moderada & 0,4 & $\begin{array}{l}\text { O fluconazol pode aumentar o risco das } \\
\text { concentrações plasmáticas do Omeprazol. }\end{array}$ \\
\hline
\end{tabular}

Fonte: Os autores.

Com base nos dados presentes no Micromedex Drug Int. foi possível classificar as interações em leve, moderada ou grave, além de citar os possíveis problemas que o paciente pode sofrer quando exposto a tais interações. As interações medicamentosas do tipo leve foram as mais frequentes $(67,1 \%)$, seguida das interações moderadas $(21 \%)$ e graves $(11,9 \%)$. As 
interações do tipo leve podem causar efeitos clínicos mínimos, enquanto que as interações moderadas podem levar a uma piora no estado clínico do neonato, e por fim as interações do tipo grave podem causar maior dano à saúde, de caráter irreversível ou que indique um risco eminente a vida. No trabalho de Mesquita $^{(23)}$ observou-se uma frequência maior de casos moderados (67\%), enquanto que neste estudo as interações do tipo leve foram mais frequentes $(67,1 \%)$

Dentre as interações medicamentosas ocorridas destacaram-se a interação entre Ampicilina e Gentamicina (52,8\%), Furosemida e Insulina (10,5\%), Ampicilina e Amicacina $(8,4 \%)$, conforme mostra a Tabela 2.

As interações mais frequentes aconteceram entre os antimicrobianos penicilinas (Ampicilina e Penicilina Cristalina) e os aminoglicosídeos (Gentamicina e Amicacina). Segundo a base de dados usada, essas interações podem ser classificadas do tipo leve, causando a diminuição da eficácia dos aminoglicosídeos pela ação das penicilinas.

A associação entre dois antimicrobianos é comum como tratamento de escolha, mas é importante ressaltar que o uso desses medicamentos deve ser feito de forma prudente, por meios de ajuste nos horários de administração de ambos os medicamentos para que os picos de concentração sejam diferentes, diminuindo a possibilidade de uma interação, mesmo do tipo leve. Para que não ocorra a resistência bacteriana é preciso o monitoramento, onde se observa a possibilidade de subdose dos aminoglicosídeos devido ao uso concomitante da penicilina ${ }^{(8,22)}$.

O uso de uma penicilina juntamente com um aminoglicosídeo faz parte do protocolo de profilaxia de sepse neonatal, de início precoce ocorrendo nas primeiras 72 horas de nascimento $^{(27)}$, o que explicaria a elevada ocorrência desta interação em UTIN Os betalactâmicos inibem a produção da parede celular da bactéria e os aminoglicosídios inibem a replicação ${ }^{(22,15)}$

A segurança de pacientes internados em UTIN é de suma importância para reduzir a morbidade e mortalidade relacionadas a erros de medicação nesses pacientes. A não identificação e prevenção de erros na utilização de medicamentos em UTIN pode levar a resultados muitos graves, com piora do quadro clínico, aumento do tempo de internação e óbito ${ }^{(25)}$. Pouco se sabe sobre os problemas relacionados ao uso de múltiplos medicamentos em neonatos, pacientes de frágil idade, que tem como defensor na sobrevida, os fármacos ${ }^{(16)}$. Neste segmento é de primordial importância a função do farmacêutico, de colaborar para a diminuição dos riscos relacionados à administração de fármacos, através do monitoramento sistemático das possíveis interações farmacológicas e manifestações de reações adversas aos fármacos ${ }^{(21,26)}$. 
A tabela 3 demonstra a frequência dos medicamentos envolvidos nos PRMs, para facilitar a comparação de dados foi usado o sistema de classificação Anatomical Therapeutic Chemical (ATC), estabelecida pela OMS, que divide as substâncias ativas em diferentes grupos, de acordo com o órgão ou o sistema que atuem e com respectivas propriedades químicas, farmacológicas e terapêuticas destas ${ }^{(28)}$.

Tabela 3. Frequência dos medicamentos envolvidos nos Problemas Relacionados aos Medicamentos de acordo com a classificação Anatomical Therapeutic Chemical(ATC)

\begin{tabular}{cc}
\hline Classe farmacêutica - ATC 1 & $\%$ \\
\hline Aparelho digestivo e metabolismo & 22,2 \\
Sangue e órgãos hematopoiéticos & 6,4 \\
Sistema cardiovascular & 10,2 \\
Hormônios de uso excluindo hormônios sexuais & 3,4 \\
Anti-infeciosos gerais para uso sistémico & 40,8 \\
Hormônios corticosteroides para uso sistémico & 0,8 \\
Sistema nervoso & 9,1 \\
Sistema respiratório & 7,1 \\
\hline
\end{tabular}

Os problemas relacionados a anti-infeciosos gerais para uso sistêmico $(40,8 \%)$ são os mais frequentes, seguido dos medicamentos utilizados para o aparelho digestivo e metabolismo $(22,2 \%)$ e sistema cardiovascular $(10,2 \%)$, o que poderia ser justificado pelo maior número de prescrições desses medicamentos em razão do perfil de criticidade dos pacientes, ressalte-se tambéma ocorrência de repetição automática da prescrição, perpetuando erros de dose, duplicidade de via de administração, dentre outros.

A etapa de prescrição é uma das fases mais críticas em termos de riscos de erro de medicação, tornando assim um ponto importante para a melhoria. $\mathrm{O}$ crescimento de formas capazes de diminuir erros requer a disposição e entendimento de suas causas e fatores $\operatorname{associados}^{(26)}$. A assistência farmacêutica no decorrer do cuidado ao paciente é essencial no ambiente hospitalar, tendo em vista que o farmacêutico também é responsável pelos resultados da terapia, aspirando evolução consistente na aplicação dos fármacos, e através disso, ser reconhecido como fração importante nos vários cenários da prática clínica ${ }^{(15)}$. 


\section{CONCLUSÃO}

Com base nos resultados encontrados, conclui-se que assegurar a segurança ao neonato abrange, no geral, o cuidado na prevenção de erros, onde o acompanhamento pelo profissional farmacêutico clínico junto a equipe multiprofissional é de suma importância, garantindo o uso seguro e racional de medicamentos. Uma vez que as consequências decorrentes do uso inadequado de medicamentos estampam um dos fundamentais fatores de perigo à segurança no cuidado.

\section{REFERÊNCIAS BIBLIOGÁFICAS}

1. Mesquita DS, Naka KS, Kawamura AS,Schmidt AS. Acolhimento de Enfermagem na Unidade de Terapia Intensiva (UTI) neonatal segundo binômio pais-filhos: estudo de revisão integrativa da literatura. Revista Eletrônica Acervo Saúde, v. 11, n. 13, p. e980-e980, 2019.

2. Ayres BV, Domingues RM, Baldisserotto ML, Leal NP, Filho FL, Caramachi AP, et al. Avaliação do local de nascimento de recém-natos com idade gestacional inferior a 34 semanas segundo complexidade da Unidade Neonatal em maternidades vinculadas à Rede Cegonha: Brasil, 2016-2017. Ciência \& Saúde Coletiva 2021.

3. Costa LD, Andersen VF, Perondi AR, França VF,Cavalheiri JC,Bortoloti DS. Fatores preditores para a admissão do recém-nascido na unidade de terapia intensiva neonatal. Revista Baiana de Enfermagem, v. 31, n. 4, 2017.

4. Queiroz KC, Nascimento MF, Fernandes V, Miotto FA. Análise de interações medicamentosas identificadas em prescrições da UTI neonatal da ICU-HGU. Journal of Health Sciences,v. 16, n. 3, 2014.

5. Carneiro AI, Silva AH. Segurança do paciente em unidade de terapia intensiva neonatal: o impacto das recomendações farmacêuticas. Conexão Fametro, 2018.

6. Conselho Federal de Farmácia. Resolução n 675 , de 31 de Outubro de 2019. Regulamenta as atribuições do farmacêutico clínico em unidade de terapia intensiva, e dá outras providências. Diário Oficial da União. 2019 nov 21 [cited 2021Maio 12]. (225 seção 1): 128. Available from: https://www.in.gov.br/web/dou/-/resolucao-n-675-de-31-de-outubro-de-2019.

7. Loureiro CV. Monitorização da farmacoterapia em recém-nascidos de extremo baixo peso em unidade de terapia intensiva neonatal. 2015.

8. Silva UD, Soeiro CL, Resque RL,Gomes MR,Costa ER,Fujishima MA, et al. Interações medicamentosas e consequentes intervenções farmacêuticas na Unidade de Terapia Intensiva de um hospital privado em Macapá, Amapá. Vigilância Sanitária em Debate: Sociedade, Ciência \& Tecnologia (Health Surveillance under Debate: Society, Science \& Technology) Visa em Debate, v. 6, n. 2, p. 29-37, 2018. 
9. Scrignoli CP, Teixeira VC, Leal DC. Interações medicamentosas entre drogas mais prescritas em unidade de terapia intensiva adulta. Revista Brasileira de Farmácia Hospitalar e Serviços de Saúde, v. 7, n. 2, 2016.

10. Silva DT,Campos CA, Vargas TG, Ziulkoski AL,Andrighetti LH,Perassolo MS. Possíveis interações medicamentosas em pacientes polimedicados de Novo Hamburgo, RS, Brasil. Infarma-Ciências Farmacêuticas, v. 30, n. 1, p. 21-29, 2018.

11. Sanitária NA, Saúde O PAN-AMERICANA, Saúde OM. A importância da farmacovigilância: monitorização da segurança dos medicamentos. 2005.

12. Paiva NA, Moura CS. Interações Medicamentosas Potenciais nas Prescrições de Pacientes Pediátricos Hospitalizados. Rev. Bras. Farm, v. 93, n. 4, p. 463-468, 2012.

13. Araújo MA,Filho WD, Silveira RS,Souza JC, Barlem EL,Teixeira NS. Segurança do paciente na visão de enfermeiros: uma questão multiprofissional. 2017.

14. Leopoldino RW. Análise dos problemas relacionados a medicamentos em Terapia Intensiva Neonatal, Ministério da Educação Universidade Federal do Rio Grande do Norte Programa de Pós-Graduação em Ciências Farmacêuticas, 2018.

15. Nunes BM,Xavier TC, Martins RR. Problemas relacinados a medicamentos antimicrobianos em unidade de terapia intensive neonatal,Revista Brasileira Terapia Intensiva,2017.

16. Costa HT. Utilização de medicamentos Off-Label e não licenciados em terapia intensiva neonatal. Dissertação do programa de pós- graduação em ciências farmacêuticas da universidade Federal do Rio Grande do Norte, 2017.

17. Pires CF. Gestão de Segurança de Pacientes Neonatos com Ênfase no Uso offlabel/unlabeled de Medicamentos - Proposta de Protocolo Terapêutico para Uso em Neonatologia.2016.

18. Nascimento J, Santos IM,Silva LJ. Cuidados com recém-nascidos alimentados por sonda gástrica: conceitos e práticas ,Universidade Federal do Estado do Rio de Janeiro, Programa de Pós-graduação em Enfermagem Rio de Janeiro, Rio de Janeiro, Brasil 2019.

19. Nascimento AR, Leopoldino RW, Santos ME,Costa TX, Martins RR. Problemas relacionados a medicamentos em neonatos cardiopatas sob terapia intensiva." Revista Paulista de Pediatria 2020.

20. Junior LA, Leite RS, Yoshida EH,Estanagel TH, Santos NS. Importância da farmácia clínica para a identificação e resolução de problemas relacionados a medicamentos (PRM).2021.

21. Silva TF,Carvalho AR. Interações medicamentosas no âmbito hospitalar e a atuação do farmacêutico nesse cenário." Revista Saúde e Desenvolvimento. 2018. 
22. Filho JM, Morais KM, Croda MT,Silva PC,Souza JM, Longhi RM. Recomendações para administração de medicamentos via sonda - Unidade de Dispensação Farmacêutica - HUUFGD/Ebserh, 2017.

23. Mesquita JC, Feitosa CL,Macêdo KP, Silva FV,Oliveira GR, Bezerra JV, et al. Análise e identificação das principais interações medicamentosas predominantes em unidade de terapia intensiva de um hospital privado." Revista Eletrônica Acervo Saúde 2020.

24. Lopes CS. Revisão de escopo sobre erros de medicação em UTI neonatal: a importância das medidas educacionais para sua prevenção. 2019.

25. Lucena MM, Freitas DE, Gomes JN, Freitas DE,Belém LF. Utilização de Medicamentos por Pacientes Neonatos e Pediátricos Em Um Hospital Filantrópico, I Congresso Brasileiro de Ciências da Saúde (CONBRACIS) 2016.

26. Hoffmeister LV,Moura GM, Macedo AP. Aprendendo com os erros: análise dos incidentes em uma unidade de cuidados neonatais." Revista Latino-Americana de Enfermagem 2019.

27. Weintraub A, Ferreira OJ, Resende LA, Filho DC,Strama MC, Gomide GP, et al .Protocolo Clínico (PC): "Condutas Médicas na Sepse Neonatal Precoce e Tardia" - Divisão Médica (DM), Uberaba, 2019.

28. Organização Mundial de Saúde (OMS). Estrutura conceitual da classificação internacional sobre segurança do doente. Direção Geral da Saúde. Lisboa, 2011. 